\title{
Circulating Tumor Cells (CTCs) as Biomarker for PD-1/PD-L1 Blockade Immunotherapy
}

Chunyan YUE ${ }^{1,2}$ and Zhiyuan HU ${ }^{1,2,3 *}$

${ }^{1}$ CAS Key Laboratory for Biomedical Effects of Nanomaterials and Nanosafety, CAS Center for Excellence in Nanoscience, National Center for Nanoscience and Technology of China, Beijing 100190, P.R. China.

${ }^{2}$ Sino-Danish College, University of Chinese Academy of Sciences, Beijing 100049, P. R. China

${ }^{3}$ Yangtze River Delta Academy of Nanotechnology and Industry Development Research, Jiaxing, Zhejiang Province, 314000, P. R. China.

\section{Description}

The PD-1/PD-L1 (programmed death-1, programmed death-1 ligand-1) checkpoint is involved in dampening autoimmunity of peripheral tissues to help control local inflammatory responses. It is reported that this pathway activation results in peripheral immunologic tolerance in T cells [1]. As an identified ligand of PD-1, PD-L1 expressed in tumor cells facilitates tumor escape by inducing a net immunosuppressive effect after binding to PD-1 present on the surface of activated $\mathrm{T}$ cells and $\mathrm{B}$ cells [2]. The enhanced understanding of the complex interplay between the tumor and the immune system has promoted the development of anti-PD therapy for the treatment of human cancers. Antibodies blocking PD-1/PD-L1 have demonstrated durable responses in a number of different advanced malignancies $[3,4]$. However, while increasing a baseline T-cell-specific immune response, immune checkpoint inhibitors might result in autoimmune-like/ inflammatory side-effects, which cause collateral damage to normal organ systems and tissues, such as skin, lung and liver [5]. Therefore, detection of potential biomarkers that may predict benefit is pivotal in order to optimize clinical efficacy and safety of checkpoint blockade immunotherapy.

Emerging data suggest that patients with PD-L1-positive tumors are more likely to benefit from PD-1/PD-L1 blockade immunotherapy than those with PD-L1-negative tumors [6-9]. Tumor-associated PDL1 immunohistochemistry (IHC) is the solely plausible biomarker to predict responses to PD-1/PD-L1 blockade and FDA has approved 4 different PD-L1 IHC assays for companion diagnostics (Table 1). However, there are multiple unresolved issues for IHC evaluation considering different IHC antibodies, primary versus metastatic biopsies, and heterogeneity of tumors. Wide variability is observed in the positive percentage in different tumor samples. Melanoma, renal cell carcinoma (RCC), non-small cell lung cancer (NSCLC) and bladder cancer are tumors classically associated with clinical responses to PD-1 checkpoint inhibition therapy, and the relevant PD-L1 IHC expression ranges widely from $14 \%$ to $100 \%$ [10-12]. It implies the possibility for PD-L1 IHC as a predictive biomarker for PD-1/PD-L1 blockade immunotherapy. In comparison, $\mathrm{PD}-\mathrm{L} 1$ expression in colorectal cancer and sarcoma ranges from $12 \%$ to $53 \%$, but the relevant responses to immune checkpoint inhibition are much weaker, indicating that tumor PD-L1 IHC expression is not the sole predictive determinant for drug response [13]. Moreover, between the matched primary tumor and distant metastasis, only a weak correlation in PD-L1 IHC detection was observed, suggesting that the primary tumor is not an adequate surrogate for PD-L1 expression detection in metastatic sites [14]. Therefore, PD-L1 detection for dynamic changes at different sites and time points will certainly be a challenge.

The analysis of blood samples for circulating tumor cells (CTCs) detection and characterization is an alternative non-invasive approach to predict response and monitor real-time response to treatment. To explore the monitor potential of CTCs during chemotherapy of metastatic breast cancer, Hartkopf et al. analyzed the correlation between changes of CTC levels and chemotherapy response assessed using radiographic Response Evaluation Criteria in Solid Tumors (RECIST) criteria and CA 15-3 concentrations. They observed that changes in CTC counts during the course of chemotherapy significantly correlated with response to chemotherapy and is useful in monitoring therapy efficacy $[15,16]$. In another study, synchronously decreases in CTC count and FLT-PET signal were found in patients undergone docetaxel therapy [17]. These proofs indicate the possibility of circulating tumor cells changes as a predicator in monitoring chemotherapy response.

Following the flourish of checkpoint blockade immunotherapy, more and more researchers are focusing on the characterization of PD-L1 on CTCs and the possibility of CTCs/PD-L1 as a biomarker for PD-1/PD-L1 blockade immunotherapy. Mazel et al. first reported the feasibility of PD-L1 evaluation in CTCs from breast cancer patients [18]. At the meeting of ASCO 2016, Boffa et al. demonstrated that patients with higher burden of PD-L1+ CTCs had a poor prognosis in lung cancer compared to those patients with low/negative PDL1+ CTCs. In metastatic bladder cancer, patients with high PD-L1+/ CD45-CTC burden and low burden of apoptotic CTCs had lower overall survival [19]. These findings suggest the possibility using PDL1 positive CTCs to evaluate prognosis in metastatic cancers. A lately study evaluated the dynamic changes of PD-L1 positive CTCs in NSCLC patients treated with PD-1 inhibitor Nivolumab. The authors correlated the status of PD-L1 positive CTCs with outcome. They found patients without PD-L1+ CTCs at 6 months of treatment all obtained a clinical benefit, while patients with PD-L1+ CTCs all experienced a disease progression. This observation indicates that PD-L1 expression on CTCs might have predictive significance in late course of anti PD-1 checkpoint immunotherapy [20]. Another latest case report has proposed that the PD-L1 assessment of CTCs might help identifying suitable patients for anti-PDL1 therapy [21]. Moreover, there are studies suggesting that PD-L1 may be induced by multiple therapies, such as cytotoxic chemotherapy, targeted agents, or radiation therapy, which may act as an immunologic escape response in tumors [22]. Therefore, assessment of PD-L1 expression on CTCs might allow serial assessment of the status of PD-L1 expression during therapy, especially

*Corresponding author: Zhiyuan HU, CAS Key Laboratory for Biomedical Effects of Nanomaterials and Nanosafety, CAS Center for Excellence in Nanoscience, National Center for Nanoscience and Technology of China, Beijing 100190, China, Tel: 861082545643; E-mail: huzy@nanoctr.cn

Received May 31, 2017; Accepted August 17, 2017; Published August 22, 2017

Citation: Chunyan YUE, Zhiyuan HU (2017) Circulating Tumor Cells (CTCs) as Biomarker for PD-1/PD-L1 Blockade Immunotherapy. Cancer Sci Ther 9: 593-594. doi: 10.4172/1948-5956.1000479

Copyright: $\odot 2017$ Chunyan YUE, et al. This is an open-access article distributed under the terms of the Creative Commons Attribution License, which permits unrestricted use, distribution, and reproduction in any medium, provided the original author and source are credited. 
Citation: Chunyan YUE, Zhiyuan HU (2017) Circulating Tumor Cells (CTCs) as Biomarker for PD-1/PD-L1 Blockade Immunotherapy. Cancer Sci Ther 9: 593-549. doi: 10.4172/1948-5956.1000479

\begin{tabular}{|c|c|c|c|}
\hline $\begin{array}{c}\text { PD-L1 detection } \\
\text { antibody }\end{array}$ & Company & $\begin{array}{r}\text { Companion } \\
\text { therapeutic agents }\end{array}$ & Histologies \\
\hline $\begin{array}{c}\text { PD-L1 IHC 28-8 } \\
\text { pharmDx }\end{array}$ & Dako & $\begin{array}{c}\text { OPDIVO (nivolumab), } \\
\text { YERVOY (ipilimumab) }\end{array}$ & $\begin{array}{c}\text { NSCLC, } \\
\text { melanoma }\end{array}$ \\
\hline $\begin{array}{c}\text { Ventana SP263 } \\
\text { PD-L1 IHC 22C3 } \\
\text { pharmDx }\end{array}$ & Roche & Imfinzi(durvalumab) & UC \\
\hline Ventana SP142 & Roche & $\begin{array}{c}\text { Keytruda } \\
\text { (pembrolizumab) }\end{array}$ & NSCLC \\
$\begin{array}{c}\text { Tecentriq } \\
\text { (Atezolizumab) }\end{array}$ & UC, NSCLC \\
\hline
\end{tabular}

Table 1: FDA approved PD-L1 IHC assays.

when repeat biopsies are impractical during the therapeutic course. Unlike traditional treatment directly targeting tumors, PD-1/PD-L1 blockade immunotherapy works via restoring immune system. The unique mechanism and the corresponding dynamic changes in PD-L1 status highlight the feasibility of PD-L1 positive CTCs as a predicator of immunotherapeutic response and a monitor biomarker for PD-1/ PD-L1 blockade therapy.

\section{References}

1. Sunshine J, Taube JM (2015) PD-1/PD-L1 inhibitors. Curr Opin Pharmacol 23 32-38.

2. Swaika A, Hammond WA, RW Joseph (2015) Current state of anti-PD-L1 and anti-PD-1 agents in cancer therapy. Mol Immunol 67: 4-17.

3. Topalian SL, Hodi FS, Brahmer JR, Gettinger SN, Smith DC, et al. (2012) (2012) Safety, activity, and immune correlates of anti-PD-1 antibody in cancer. N Engl J Med 366: 2443-2454.

4. Brahmer JR, Tykodi SS, Chow LQ, Hwu WJ, Topalian SL, et al. (2012) Safety and activity of anti-PD-L1 antibody in patients with advanced cancer. $\mathrm{N}$ Engl J Med 366: 2455-2465.

5. Postow MA (2015) Managing immune checkpoint-blocking antibody side effects. Am Soc Clin Oncol Educ Book 76-83.

6. Fehrenbacher L, Spira A, Ballinger M, Kowanetz M, Vansteenkiste J, et al. (2016) Atezolizumab versus docetaxel for patients with previously treated non-small-cell lung cancer (POPLAR): A multicentre, open-label, phase 2 randomised controlled trial. Lancet 387: 1837-1846.

7. Herbst RS, Baas P, Kim DW, Felip E, Pérez-Gracia JL, et al. (2016) Pembrolizumab versus docetaxel for previously treated, PD-L1-positive, advanced non-small-cell lung cancer (KEYNOTE-010): A randomised controlled trial. Lancet 387: 1540-1550.

8. Gandini S, Massi D, Mandalà M (2016) PD-L1 expression in cancer patients receiving anti PD-1/PD-L1 antibodies: A systematic review and meta-analysis. Crit Rev Oncol Hematol 100: 88-98.

9. Abdel-Rahman O (2016) Correlation between PD-L1 expression and outcome of NSCLC patients treated with anti-PD-1/PD-L1 agents: A meta-analysis. Crit Rev Oncol Hematol 101: 75-85.

10. Dong H, Strome SE, Salomao DR, Tamura H, Hirano F, et al. (2002) Tumorassociated B7-H1 promotes T-cell apoptosis: a potential mechanism of immune evasion. Nat Med 8: 793-800.

11. Powles T, Eder JP, Fine GD, Braiteh FS, Loriot Y, et al. (2014) MPDL3280A (anti-PD-L1) treatment leads to clinical activity in metastatic bladder cancer. Nature 515: 558-562.

12. Herbst RS, Soria JC, Kowanetz M, Fine GD, Hamid O, et al. (2014) Predictive correlates of response to the anti-PD-L1 antibody MPDL3280A in cancer patients. Nature 563-567.

13. Herbst RS, Soria JC, Kowanetz M, Fine GD, Hamid O, et al. (2014) Predictive correlates of response to the anti-PD-L1 antibody MPDL3280A in cancer patients. Nature 563-567.

14. Jilaveanu LB, Shuch B, Zito CR, Parisi F, Barr M, et al. (2014) PD-L1 expression in clear cell renal cell carcinoma: An analysis of nephrectomy and sites of metastases. J Cancer 5: 166-172.

15. Alix-Panabières $\mathrm{C}$, Schwarzenbach $\mathrm{H}$, Pantel $\mathrm{K}$ (2012) Circulating tumor cells and circulating tumor DNA. Annu Rev Med 63: 199-215.

16. Hartkopf AD, Wagner $P$, Wallwiener D, Fehm $T$ (2011) Changing levels of circulating tumor cells in monitoring chemotherapy response in patients with metastatic breast cancer. Anticancer Res 31: 979-984.

17. Contractor K, Aboagye EO, Jacob J, Challapalli A, Coombes RC, et al. (2012) Monitoring early response to taxane therapy in advanced breast cancer with circulating tumor cells and [(18) F] 3 -deoxy-3 -fluorothymidine PET: a pilot study. Biomark Med 6: 231-233

18. Mazel M, Jacot W, Pantel K, Bartkowiak K, Topart D, et al. (2015) Frequent expression of PD-L1 on circulating breast cancer cells. Mol Oncol 9: 17731782.

19. Anantharaman A, Friedlander T, Lu D, Krupa R, Premasekharan G (2016) Programmed death-ligand 1 (PD-L1) characterization of circulating tumor cells (CTCs) in muscle invasive and metastatic bladder cancer patients 16: 744

20. Nicolazzo C, Raimondi C, Mancini M, Caponnetto S, Gradilone A, et al. (2016) Monitoring PD-L1 positive circulating tumor cells in non-small cell lung cancer patients treated with the PD-1 inhibitor Nivolumab. Sci Rep 6: 31726.

21. Kulasinghe A, Perry C, Kenny L, Warkiani ME, Nelson C, et al. (2017) PD-L1 expressing circulating tumour cells in head and neck cancers 17: 333.

22. Adams DL, Adams DK, He J, Kalhor N, Zhang M, et al (2017) Sequential tracking of PD-L1 expression and RAD50 induction in circulating tumor and stromal cells of lung cancer patients undergoing radiotherapy. Clin Cancer Res. 